\title{
Etude Des Anomalies De Résistivités Pour L'optimisation Des Implantations De Forages D'hydraulique Villageoise En Milieu De Socle: Cas De La Région Du Haut-Sassandra (Centre-Ouest De La Côte d'Ivoire)
}

Kouassi Kouamé Auguste, M.A. Kouassi Francis Williams, M.A.

Université Nangui Abrogoua, UFR Sciences et Gestion de l'Environnement, Laboratoire Géosciences et Environnement, Abidjan, Côte d'Ivoire.

Kouadio Zilé Alex, M.A.

Université Jean Lorougnon Guedé Daloa, UFR Environnement, Daloa, Côte d'Ivoire.

\section{Ballo Bi Herber Esaïe, Student}

Université Nangui Abrogoua, UFR Sciences et Gestion de l'Environnement, Laboratoire Géosciences et Environnement, Abidjan, Côte d'Ivoire.

Coulibaly Adama, A.

Département des Sciences et Techniques de l'Eau et du Génie de l'Environnement, UFR des Sciences de la Terre et des Ressources Minières, Université Félix Houphouët-Boigny, Abidjan, Côte d'Ivoire

Kamagaté Bamory, M.C.

Université Nangui Abrogoua, UFR Sciences et Gestion de l'Environnement, Laboratoire Géosciences et Environnement, Abidjan, Côte d'Ivoire.

Doi: 10.19044/esj.2018.v14n24p69 URL:http://dx.doi.org/10.19044/esj.2018.v14n24p69

Abstract

Effective groundwater collecting in the basement requires characterization and identification of the productive aquifer systems that are developed there. Therefore, our study relates to the identification, analysis and description of success indicators of geophysical implantations. It about the type and form of anomaly and the type of survey curve. These indicators are obtained following interpretation of trainings and electrical soundings data. A statistical analysis of these indicators is done using the crosstab method. This reveals that drilling productivity is influenced by indicators mentioned above. Indeed, the combination of type A or $\mathrm{H}$ soundings obtained straight up CCL type and $\mathrm{W}$ or $\mathrm{U}$ form anomalies has the best results of up to $100 \%$ success rate. The flow rates obtained are generally large $\left(\mathrm{Q}>4 \mathrm{~m}^{3} / \mathrm{h}\right)$. On the other 
hand, drilling implanted from a type A sounding on a $\mathrm{U}$ or $\mathrm{V}$ form anomaly and CCE type has high failure rates (33\% to 60\%).

Keywords: Indicators, Implantations, Optimization, Drilling, Base, UpperSassandra

\section{Résumé}

Le captage efficient des eaux souterraines en milieu de socle nécessite de caractériser et d'identifier les systèmes aquifères productifs qui y sont développés. Notre étude porte donc sur l'identification, l'analyse et la description d'indicateurs de succès des implantations géophysiques. Il s'agit du type et de la forme de l'anomalie et du type de courbe de sondage. Ces indicateurs sont obtenus suite à l'interprétation des données de trainés et de sondages électriques. Une analyse statistique de ces indicateurs est faite à l'aide de la méthode du tableau croisé. Celle-ci révèle que la productivité du forage est influencée par les indicateurs sus cités. En effet, la combinaison des sondages de type $\mathrm{A}$ ou $\mathrm{H}$ obtenus à l'aplomb des anomalies de type CCL et de forme $\mathrm{W}$ ou $\mathrm{U}$ présente les meilleurs résultats allant jusqu'à $100 \%$ de taux de succès. Les débits obtenus sont dans l'ensemble importants $(\mathrm{Q}>4$ $\mathrm{m}^{3} / \mathrm{h}$ ). Par contre, les forages implantés à partir d'un sondage de type A obtenu sur une anomalie de forme $\mathrm{U}$ ou $\mathrm{V}$ et de type CCE ont des taux d'échec élevés (33\% à 60\%).

Mots clés : Indicateurs, Implantations, Optimisation, Forages, Socle, HautSassandra

\section{Introduction}

Selon les travaux réalisés par Amani et al. (2013) dans la Souspréfecture d'Angoda (Toumodi), les disfonctionnements (le dépérissement et l'intermittence des forages) des pompes à motricité humaine $(\mathrm{PMH})$ sont dus pour la plupart à leur mauvaise implantation dans 57\% des cas. Or, les forages captant les fractures généralement bien alimentées et capables de fournir des débits assez importants sont aujourd'hui les plus sûrs et les moins influençables par les fluctuations saisonnières (Biémi, 1992), et donc à l'abri du dépérissement ou de l'intermittence. Pour une meilleure identification des ressources souterraines, il est donc nécessaire de localiser les systèmes aquifères développés en milieux de socle cristallin et cristallophyllien. C'est dans cette optique que s'inscrit cette étude dont le thème est «Etude d'indicateurs géophysiques pour l'optimisation des implantations de forages d'hydraulique villageoise en milieu de socle : cas de la région du HautSassandra (centre-ouest de la Côte d'Ivoire) ». L'objectif est donc d'identifier des indicateurs géophysiques de succès d'implantation des forages. 


\section{Présentation de la zone d'étude}

La région du Haut-Sassandra est située au Centre-Ouest de la Côte d'Ivoire entre $6^{\circ} 05^{\prime}$ et $6^{\circ} 55^{\prime}$ de longitude Ouest et entre $6^{\circ} 02^{\prime}$ et $7^{\circ} 55^{\prime}$ de latitude Nord. Elle se compose des départements de Vavoua au nord, de Daloa au centre et d'Issia au sud (Figure 1).

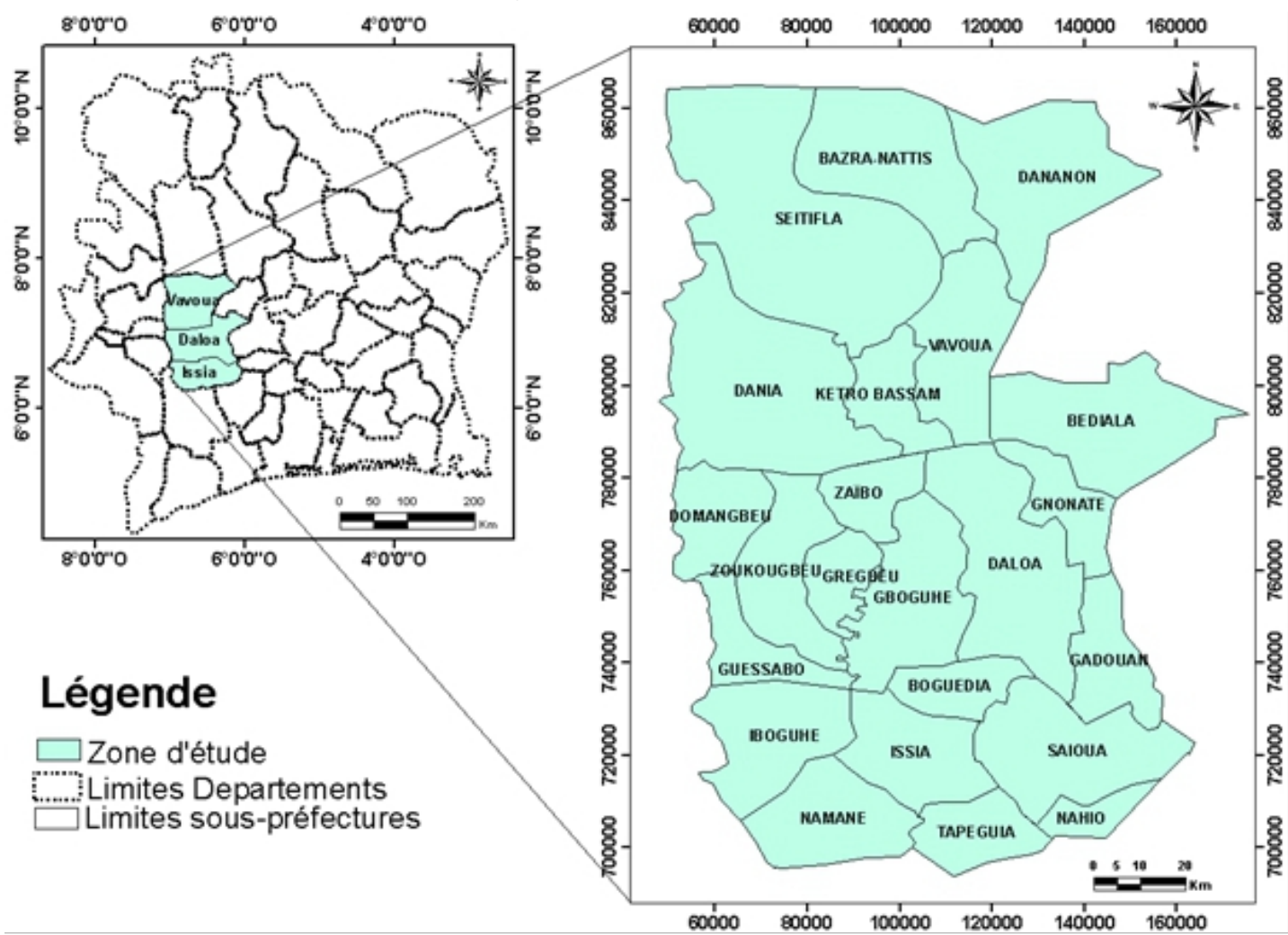

Figure 1 : Présentation de la zone d'étude

Cette région appartient au climat tropical humide avec une pluviométrie oscillant entre 1200 et 1600 millimètres de hauteur de pluie par an (SODEXAM, 2010).

Le sol est ferralitique d'origine granitique moyennement à faiblement dénaturés. A côté des sols ferralitiques, les classes de sols les plus représentées sont les sols peu évolués (d'apport alluvial, et/ou colluvial) et les sols hydromorphes (Koffi, 2013).

Les formations géologiques rencontrées dans la région comprennent les roches magmatiques et métamorphiques. Les roches magmatiques sont représentées essentiellement par les granitoïdes qui se rencontrent pratiquement dans la majeure partie de la région. Elles se composent des granites et des granodiorites. Les roches métamorphiques du bassin quant à elles, sont de type migmatites et schisteux. Les faciès migmatitiques 
rencontrés dans la zone d'étude sont constitués par les migmatites anciennes, les migmatites rubanées et les leucomigmatites (Bonnot, 1985 in Ballo, 2016). L'ensemble schisteux comprend les schistes et de roches vertes.

Du point de vue hydrogéologique, le modèle équivalent à la zone d'étude est le modèle conceptuel stratiforme de la structure hydrogéologique des aquifères de socle proposé par Lachassagne et Wyns (2005). Il a la stratigraphie suivante : cuirasse, altérites, horizon fissuré et roche saine. A l'instar des autres régions situées en milieu cristallin et le cristallophyllien, les systèmes d'aquifères rencontrés sont constitués d'aquifères d'altérites et d'aquifères de fissures.

Dans la plupart des cas, l'ensemble altérites plus horizon fissuré constitue un aquifère composite qui fonctionne comme un tout, surtout lorsqu'il est exploité au moyen de forages (Dewandel et al., 2006).

Les aquifères de fissures sont des roches dures qui présentent un comportement mécanique et des propriétés hydrauliques d'ensemble relativement homogènes et qui se caractérisent principalement par une perméabilité de fissures ou de fractures (Lachassagne et Wyns, 2005). Les nappes qu'elles renferment sont considérées comme "discontinues", en raison de l'importante variabilité spatiale de leurs propriétés hydrodynamiques (Lachassagne et Wyns, 2005). Ces aquifères regorgent une importante ressource en eau et assurent la fonction transmissive de l'aquifère composite. C'est lui qui est capté dans la plupart des forages des régions de socle, qui sont en général verticaux (Lachassagne et al., 2005).

\section{Matériel et Méthodes Données}

Les données hydrogéologiques (log des forages issus des implantations géophysiques et géomorphologiques) utilisées ont été collectées auprès de l'Office National de l'Eau Potable (ONEP) dans le cadre du programme d'hydraulique village Café-Cacao 2014. L'échantillonnage a concerné 13 forages implantés suivant la géophysique dans 11 localités. Dans 8 autres localités, l'échantillonnage a concerné 11 forages implantés selon les indicateurs géomorphologiques (Figure 2). L'ensemble des 24 forages a été réalisé dans des formations granitiques.

Les données géophysiques concernent les résistivités électriques obtenues par les techniques de trainé et de sondage électriques, à l'aide du résistivimètre Syscal R2. 


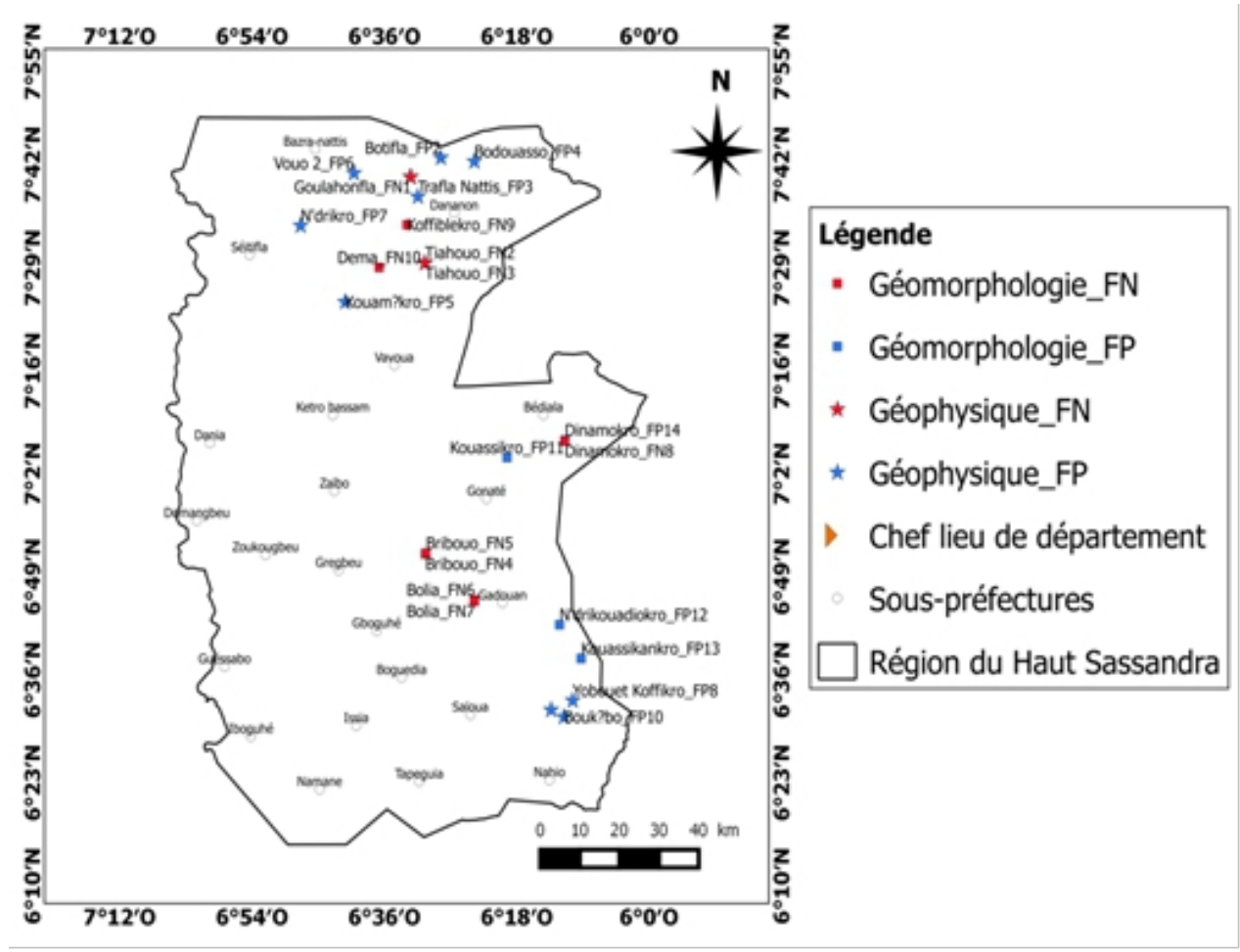

Figure 2 : Réseau des forages réalisés

\section{Méthodologie}

\section{Acquisition des données de résistivité}

Deux types de mesures électriques ont été réalisés : le trainé et le sondage électrique.

Les levés de traîné électrique permettent une investigation latérale en vue de déterminer les discontinuités du socle. Sa mise en œuvre a consiste à déplacer sur le terrain, le long de layons, le quadripôle AMNB de dimension fixe ( $\mathrm{AB}$ environ 300 et $450 \mathrm{~m}$ ) parallèlement à lui-même : il s'agit du dispositif wenner. A chaque station, on mesure l'intensité de courant $\mathrm{I}$, la différence de potentiel $\Delta \mathrm{V}$ et on calcul la résistivité apparente $\left(\rho_{\mathrm{a}}\right)$ qu'on affecte au centre $\mathrm{O}$ du dispositif. Ceci permet une profondeur d'investigation à peu près constante.

A la suite des trainés électriques, sont mis en œuvre les sondages électriques. Ces derniers sont réalisés au niveau des anomalies conductrices révélées par les trainés. La technique du sondage électrique permet d'obtenir des informations quantitatives sur les variations de la résistivité avec la profondeur. Le dispositif utilisé dans le cadre de cette étude est le dispositif Schlumberger. Sa mise en œuvre nécessite l'emploi de quatre électrodes (A, $\mathrm{B}, \mathrm{M}$ et $\mathrm{N}$ ) qui sont méthodiquement écartées du point central (centre du sondage) au fur et à mesure afin de prospecter une tranche de terrains de plus 
en plus grande. On exécute en un même endroit une succession de mesures, en augmentant chaque fois les dimensions du dispositif (écartement entre les électrodes A et B) et de ce fait la profondeur d'investigation. La distance MN quant à elle, est maintenue fixe pendant un certain nombre de mesures.

\section{Interprétation des données de résistivités}

Le traitement des données de trainé électrique est fait avec Microsoft EXCEL. Il s'agit de construire suivant une échelle sémi-logarithmique des profils de résistivités apparentes à partir desquelles l'on discrimine les types et formes d'anomalies conductrices. Au droit de ces anomalies ont été exécutés des sondages électriques.

Le traitement des données de sondage est fait avec le logiciel IPI2WIN qui a consisté à la représentation et à l'interprétation des courbes de sondages électriques. Les valeurs de résistivités apparentes sont reportées sur une échelle bi-logarithmique avec en abscisse, les distances $\mathrm{AB} / 2$. L'interprétation avec ce logiciel utilise des algorithmes d'inversion. Elle consiste à comparer les courbes pré-calculées pour divers modèles (épaisseur et résistivité variables) avec des résultats de terrain (courbes de sondage électrique) (Figure 3). Le but de l'opération est de créer un modèle du sous-sol constitué d'une succession de couches horizontales ayant chacune une certaine résistivité et une certaine épaisseur (B) puis de calculer la réponse de ce modèle (C) (Chapellier, 2000 ; 2001 ; Marescot, 2009).

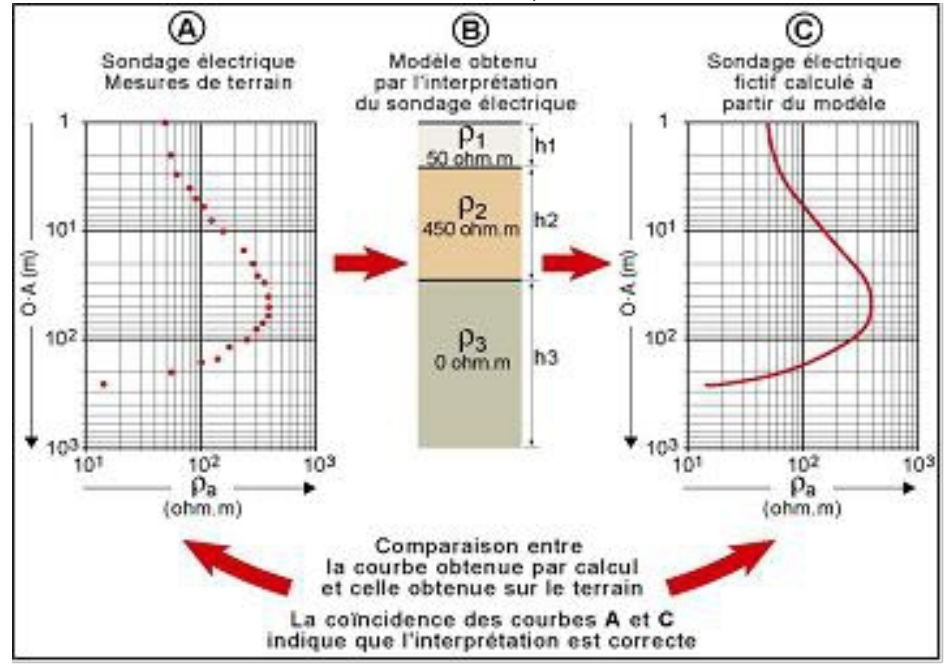

Figure 3 : Interprétation automatique de données de sondage (Marescot, 2009)

\section{Analyse corrélative des données}

Le tableau croisé est la méthode utilisée pour le traitement statistique de nos données. Il présente l'avantage d'être l'une des formes de structuration des données les plus courantes dans l'analyse des variables qualitatives. Il 
permet en effet de travailler sur des variations par catégorie d'éléments indispensables en vue d'une interprétation des résultats. L'étude concerne trois variables qualitatives à savoir : «le type d'anomalie», « la forme d'anomalie » et « le type de sondage ». Par cette méthode, il s'agira d'abord de déterminer les fréquences des différentes modalités des variables et ensuite, de mettre en évidence les corrélations éventuelles entre ces modalités. La première approche consiste à déterminer les paramètres géophysiques induisant la productivité des forages, la deuxième consiste à identifier la combinaison des indicateurs «type d'anomalie », « forme d'anomalie », et «type de sondage » conduisant à un taux élevé des forages positifs.

Enfin, pour mieux appréhender la concordance entre ces résultats géophysiques et ceux de forage, une étude corrélative est faite. Il s'est agi de confronté pour chaque couple (SE ; F), les paramètres suivants : le nombre et la nature des couches géologiques et leur épaisseur correspondant.

\section{Résultats}

\section{Identification des indicateurs de succès de forage}

\section{Types d'anomalies conductrices et taux de succès}

Les types d'anomalies les plus représentatifs dans l'effectif étudié dans la région sont: Compartiment Conducteur Etroit (CCE), Compartiment Conducteur Large (CCL) et Contact Entre Deux Palier (CEDP) avec respectivement $54 \%, 38 \%$ et $8 \%$. L'anomalie de type Palier Conducteur (PC) est par contre inexistant (Figure 4).

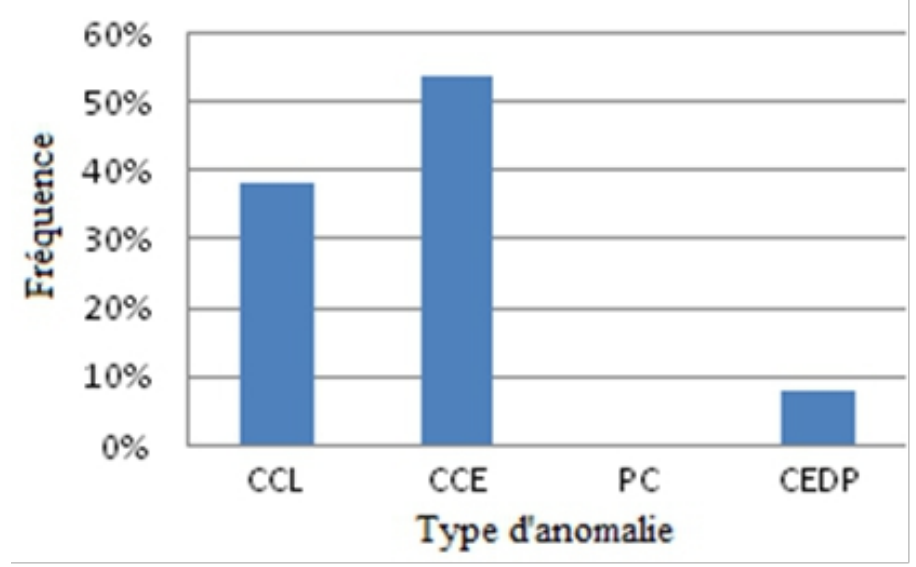

Figure 4: Fréquence des types d'anomalies

Concernant les taux de succès des forages, les types d'anomalies CCL et CEDP sont satisfaisants à $100 \%$ alors que le type CCE ne l'est qu'à 53\% (Figure 5). 


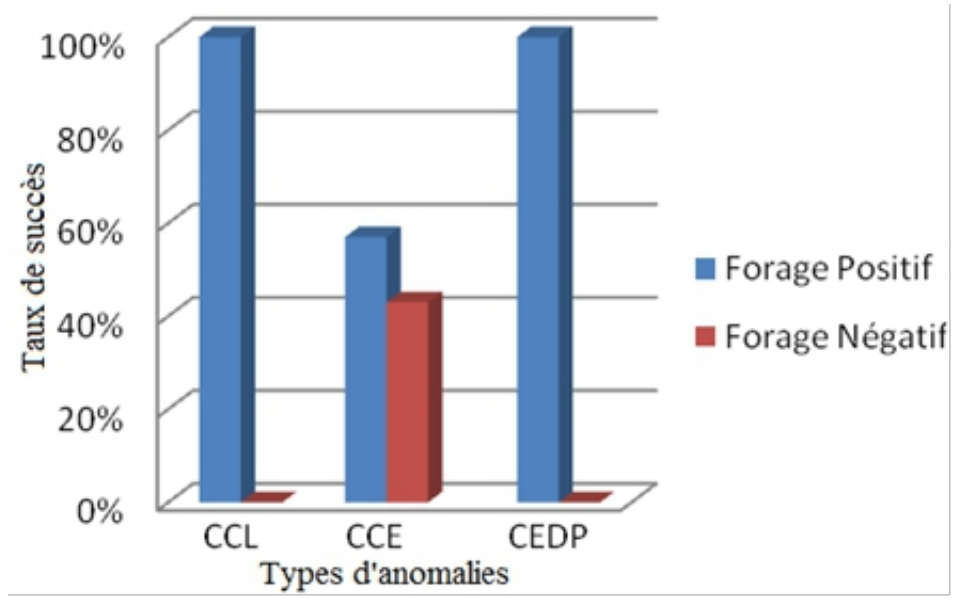

Figure 5: Taux de succès par type d'anomalie

\section{Formes d'anomalies conductrices et taux de succès}

Les formes d'anomalies $\mathrm{U}, \mathrm{V}$ et $\mathrm{W}$ sont les plus représentatifs avec des proportions respectives de $29 \%, 35 \%$ et $24 \%$ (Figure 6). Quant à la forme d'anomalie K, elle est peu rencontrée dans l'effectif étudié (12\%).

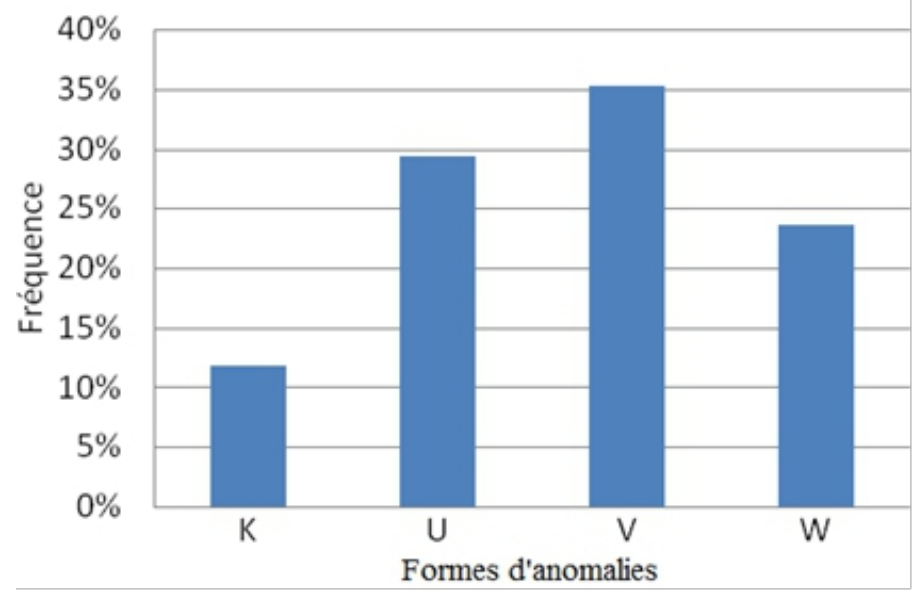

Figure 6: Fréquence des formes d'anomalies

Le taux de succès correspondant aux formes d'anomalies $\mathrm{K}, \mathrm{U}, \mathrm{V}$ et $\mathrm{W}$ présenté à la figure 7 , montre que les formes $\mathrm{K}$ et $\mathrm{W}$ ont un taux de succès de $100 \%$. Ensuite suivent les formes $\mathrm{U}$ et $\mathrm{V}$ avec des taux respectifs de $80 \%$ et $50 \%$. 


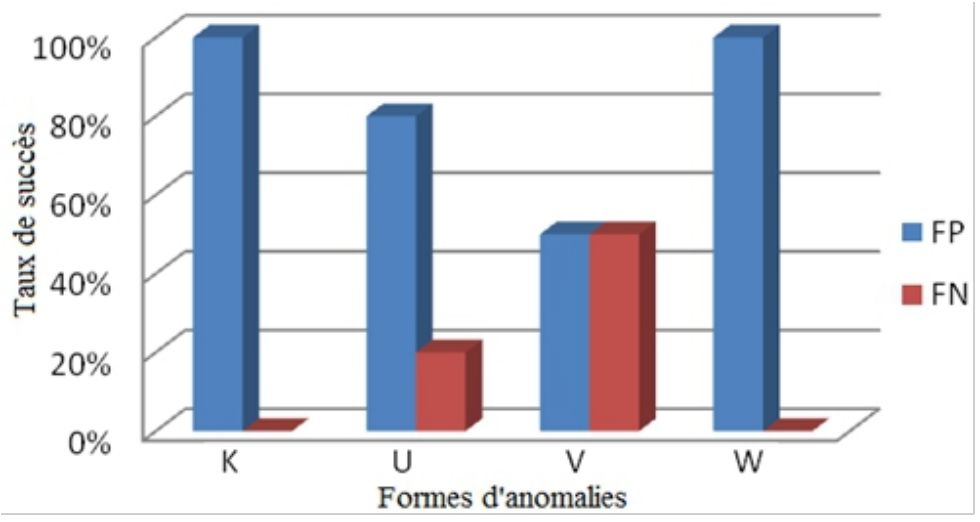

Figure 7: Taux de succès en fonction de la forme d'anomalie

Au regard de qui précède, il apparait clair qu'il existe une relation entre la forme de l'anomalie et la productivité du forage. Ainsi, la forme W est plus favorable à la réalisation de forages positifs car représentative et satisfaisante à $100 \%$. A l'opposé, la forme $\mathrm{V}$ est moins favorable à l'implantation du forage dans la zone d'étude.

\section{Corrélation entre types et formes d'anomalies conductrices selon le taux de succès}

La figure 8 indique que les anomalies de types CCE sont les seuls à enregistrer un taux d'échec de $60 \%$ pour les anomalies de forme $\mathrm{V}$ et de $33 \%$ pour celles de forme U. A l'opposé, les anomalies de types CCL de forme $\mathrm{U}$ et $\mathrm{W}$, et celles de type CEDP et de forme $\mathrm{K}$, s'avèrent satisfaisantes avec un taux de succès de $100 \%$.

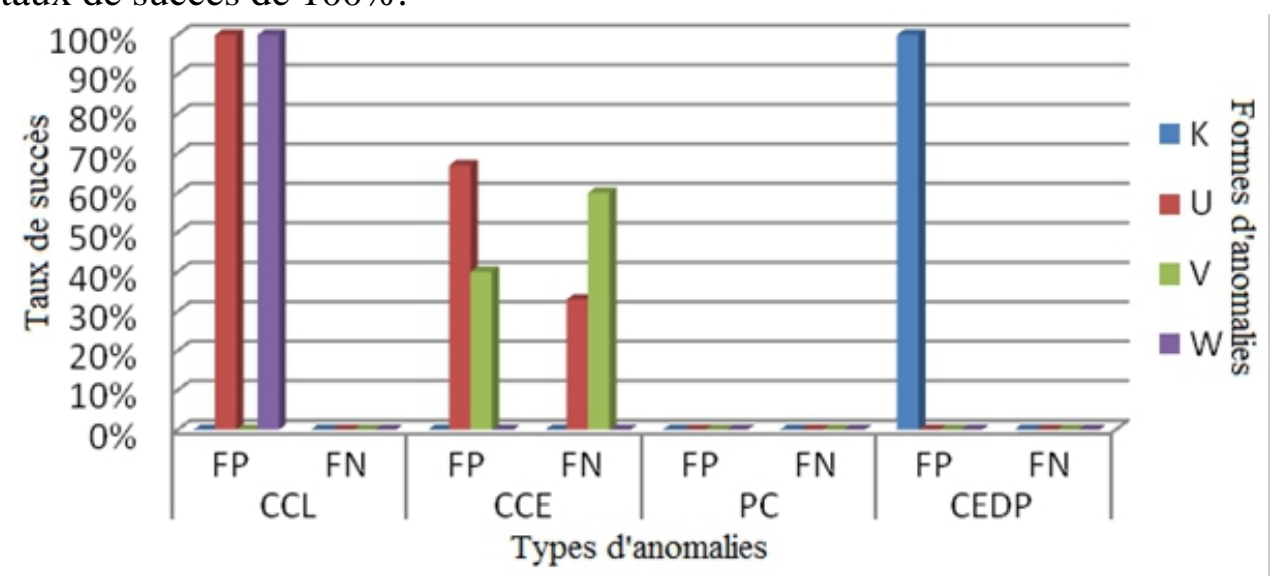

Figure 8: Taux de succès en fonction du type et de la forme d'anomalie

\section{Types de sondages électriques}

Le diagramme de distribution de la figure 9, indique que les courbes de sondages couramment rencontrés dans la zone d'étude sont d'abord de 
types A avec une fréquence de $69 \%$. Ils sont suivis des courbes de sondages de types $\mathrm{H}(23 \%)$ et enfin de ceux de types $\mathrm{KH}$ avec $8 \%$ de fréquence.

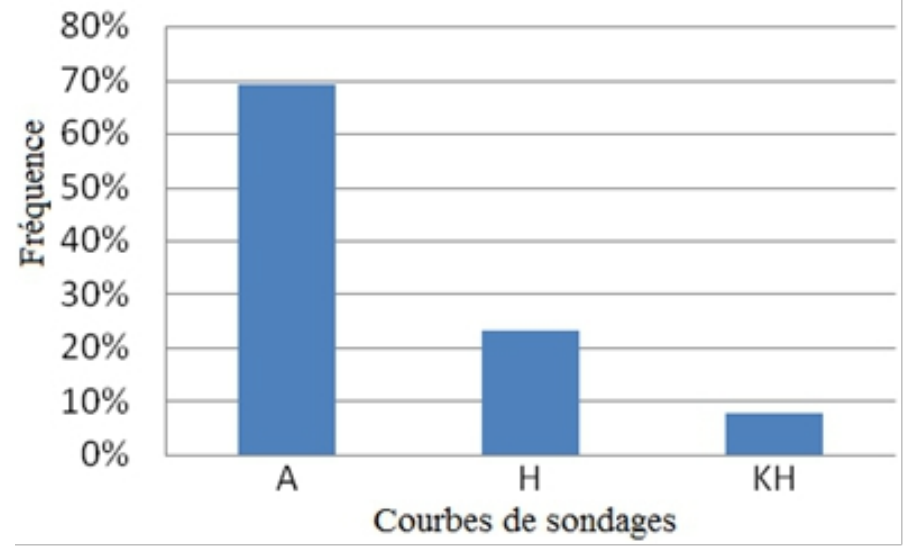

Figure 9: Fréquence des courbes de sondages

\section{Taux de succès selon le type d'anomalie et le type de sondage}

Quelque soit le type de courbe de sondage (A, H et $\mathrm{KH})$, le taux de succès est de $100 \%$ lorsque ces sondages ont été réalisés sur des anomalies de type CCL. Aussi, pour les courbes de sondages de type H et A, ce même taux est-il obtenu au niveau des anomalies de type CCE et CEDP. Par contre, au niveau des anomalies de type $\mathrm{CCE}$, les sondages de type A rencontrés présentent un taux d'échec de 60\% (Figure 10).

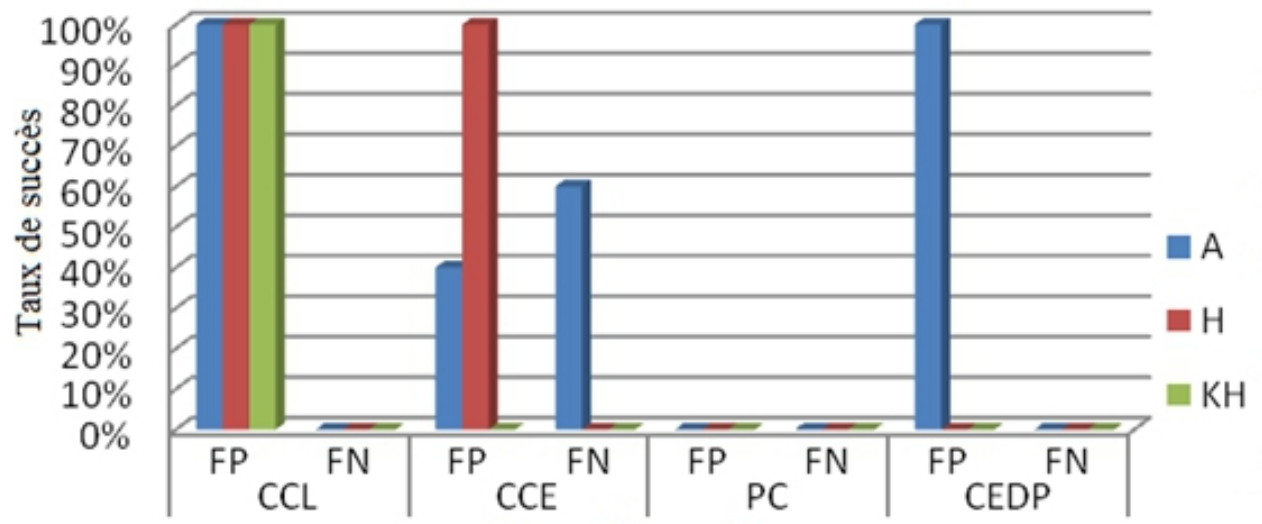

Types d'anomalies

Figure 10: Taux de succès en fonction des types d'anomalie et de courbes de sondage

Taux de succès selon la forme d'anomalies conductrices et le type de sondage

Selon la Figure 11, le taux de succès est de :

- $100 \%$ pour les sondages de types A et $\mathrm{H}$ rencontrés sur les formes d'anomalies K, 
- $75 \%$ pour les sondages de types A et $100 \%$ pour les types $\mathrm{H}$ et KH rencontrés sur les formes d'anomalies U,

- également $100 \%$ pour les sondages de types A rencontrés sur les formes d'anomalies W,

- environ $33 \%$ pour les sondages de types A rencontrés sur les formes d'anomalies $\mathrm{V}$.

Seuls des forages négatifs ont été obtenus pour des courbes de sondage de type A réalisés sur des formes d'anomalies $\mathrm{U}$ et $\mathrm{V}$.

De cette analyse, il ressort que les sondages de types A, sur les anomalies de formes $\mathrm{W}$ et $\mathrm{K}$ et les sondages de types $\mathrm{H}$ sur anomalies de formes $\mathrm{K}$ et $\mathrm{U}$ favorisent un taux de succès élevé. Par contre, les sondages de types A sur des anomalies de formes $\mathrm{V}$ induisent un taux de forage négatif élevé.

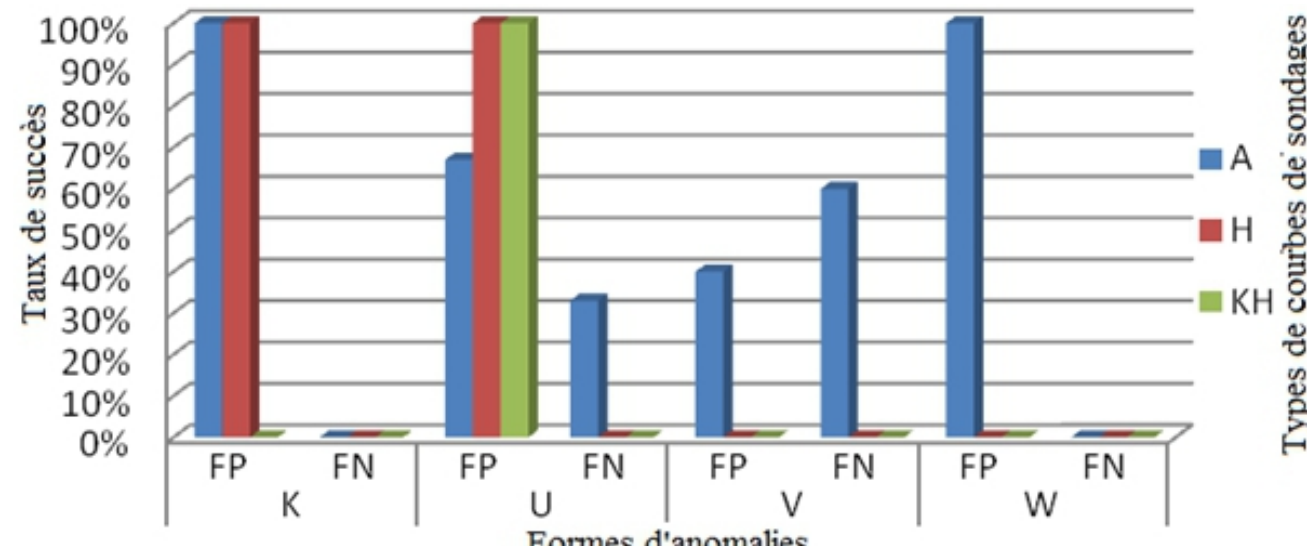

Figure 11: Taux de succès en fonction des formes d'anomalie et types de courbes de sondage

\section{Synthèse sur les indicateurs de succès de forage}

Le récapitulatif des différentes combinaisons d'indicateurs (types d'anomalies, formes d'anomalies et types de sondages) avec leurs taux de succès est résumé dans le tableau I.

Tableau I: Synthèse des indicateurs géophysiques de succès d'un forage

\begin{tabular}{ccccc}
\hline TYPES & FORMES & TYPES DE & TAUX DE & TAUX \\
D'ANOMALIES & D'ANOMALIES & SONDAGES & SUCCES & D'ECHEC \\
\hline CCL & $\mathrm{W}$ & $\mathrm{A}$ & $100 \%$ & $0 \%$ \\
& $\mathrm{U}$ & $\mathrm{H}$ & $100 \%$ & $0 \%$ \\
$\mathrm{CEDP}$ & $\mathrm{K}$ & $\mathrm{KH}$ & $100 \%$ & $0 \%$ \\
& & $\mathrm{~A}$ & $100 \%$ & $0 \%$ \\
$\mathrm{CCE}$ & $\mathrm{H}$ & $100 \%$ & $0 \%$ \\
& $\mathrm{U}$ & $\mathrm{A}$ & $67 \%$ & $33 \%$ \\
& $\mathrm{~V}$ & $\mathrm{~A}$ & $40 \%$ & $60 \%$ \\
\hline
\end{tabular}


L'analyse du tableau révèle que l'optimisation de l'implantation d'un forage par la méthode de résistivité électrique repose sur la combinaison d'indicateurs qualitatifs. Ainsi, un sondage de type A ou H, sur une anomalie de forme $\mathrm{W}$ ou $\mathrm{U}$ et de type $\mathrm{CCL}$, caractérise les forages à taux de succès allant jusqu'à $100 \%$. Egalement, les sondages de type $\mathrm{KH}$, sur les anomalies de forme $\mathrm{U}$ et de type CCL, puis les sondages de type A ou $\mathrm{H}$ sur des anomalies de formes $\mathrm{K}$ et de type CEDP présentent un taux de succès atteignant 100\%. Par contre, les forages implantés à partir d'un sondage de type A obtenu sur une anomalie de forme $\mathrm{U}$ ou $\mathrm{V}$ et de type CCE ont des taux d'échec élevés (33\% à 60\%).

\section{Analyse comparative des résultats géophysiques et logs de forage Cas de forages négatifs}

Les anomalies repérées dans les localités de Goulahonfla et Tiahouo sont de type Compartiment Conducteur Etroit (CCE) et de forme V. Les sondages réalisés à l'aplomb de ces anomalies sont de type A. Toutefois, ces dernières présentent quelques différences.

L'interprétation des courbes de sondage SE1 de Goulahonfla indique quatre couches avec une épaisseur d'altération estimée à 19,85 m. Les deux premières couches d'une épaisseur de $8,5 \mathrm{~m}$, se composent de la terre arable ou végétale et des argiles. En dessous, l'on trouve la troisième couche, de faible résistivité $(47,9 \Omega . \mathrm{m})$ correspondant à la zone altérée juste au-dessus du socle sain. Enfin, le dernier niveau (socle sain), qui se caractérise par une forte résistivité et par une remontée brusque de la courbe avec une pente supérieure à $45^{\circ}$ caractéristique du socle cristallin, se situe à une profondeur d'environ 20 $\mathrm{m}$ (Figure 12a). 


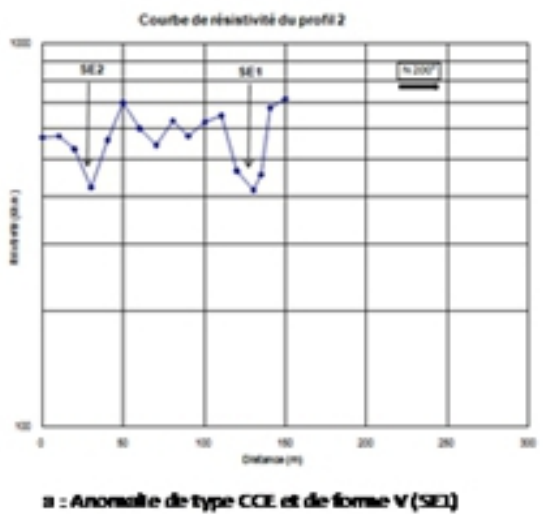

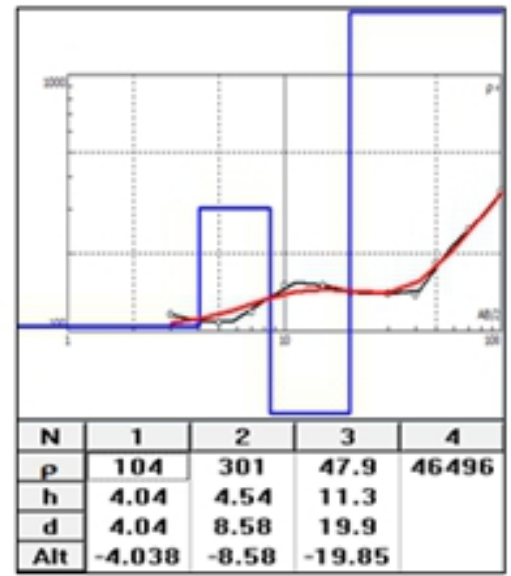

b:SondzedetypeA

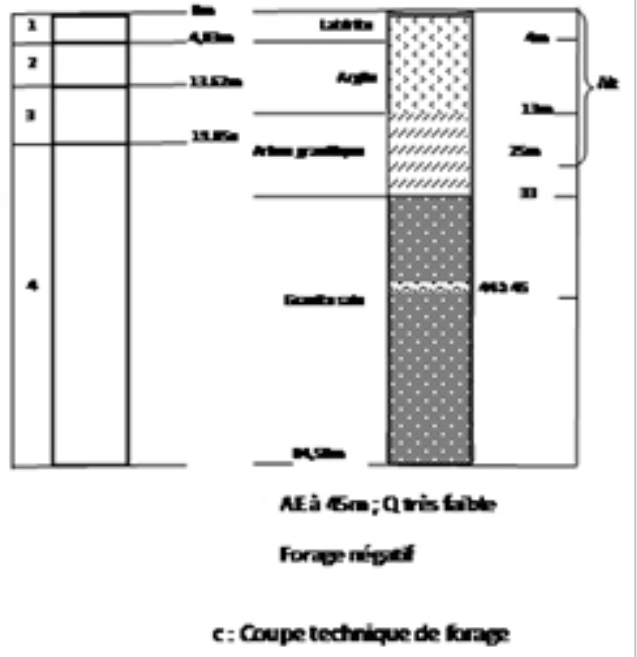

Figure 12 a : Corrélation entre les données géophysiques et les forages négatifs : cas de Goulahonfla

Quant au log de forage, il présente une lithologie de 4 couches constitués d'abord de cuirasse latéritique, ensuite d'argile, puis d'arène granitique et enfin de granite sain. Le forage réalisé est profond de $84,5 \mathrm{~m}$ avec une altération de $25 \mathrm{~m}$ et une humidité constatée à $44 \mathrm{~m}$. Il a été déclaré négatif. A Tiahouo, le sondage $S E$ présente quatre couches avec une allure à remontée trainante quasi-horizontal et monotone entre $\mathrm{AB} / 2$ allant de 15 à $100 \mathrm{~m}$ de profondeur. L'épaisseur des trois premières couches est estimée à $68 \mathrm{~m}$. Au niveau du log de forage, on a aussi quatre couches à savoir : la cuirasse latéritique, l'argile, le granite altéré et le granite sain (Figure 12b). Le forage réalisé est profond de $99,20 \mathrm{~m}$ avec $25,20 \mathrm{~m}$ d'altération et une humidité constatée à $43 \mathrm{~m}$. 


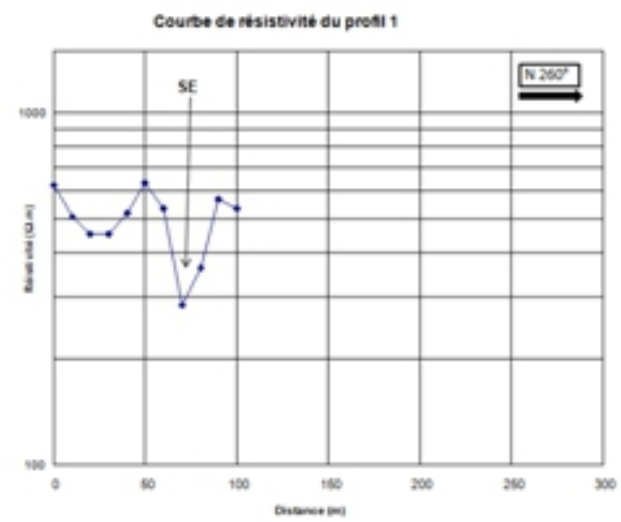

d : Anomelie de type CCE et deformev

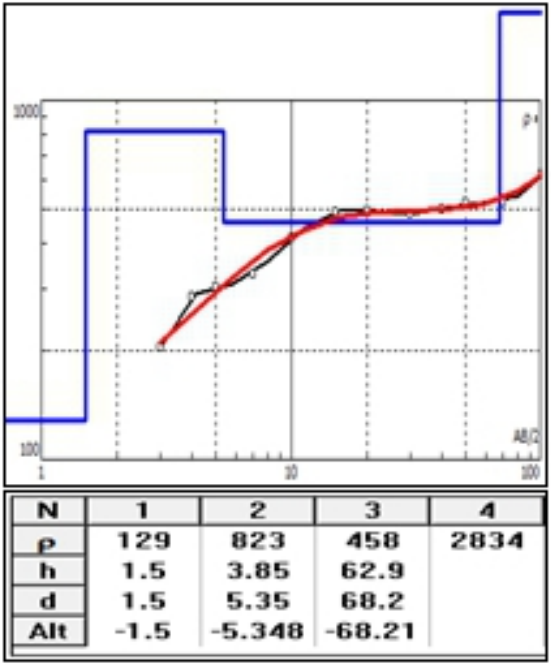

E : Sondage detype A

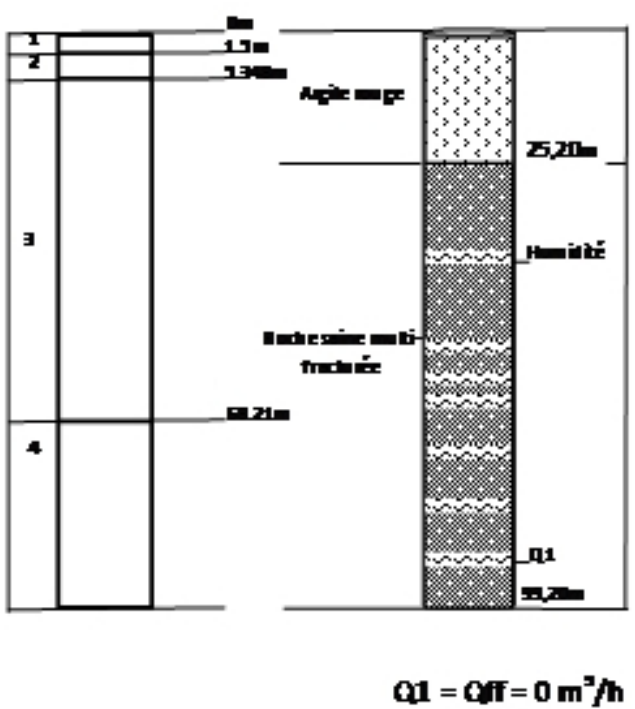

Forage négatif

\section{f: Coupe technique de forage}

Figure 12 b: Corrélation entre les données géophysiques et les forages négatifs : cas de Tiahouo

\section{Cas de forages positifs}

La bonne combinaison des indicateurs géophysiques a conduit à un taux élevé de forages positifs dans plusieurs localités.

A Kouamékro, un sondage de type $\mathrm{H}$ a été obtenu à l'intersection de deux trainés d'orientation $\mathrm{N} 00^{\circ}$ et $\mathrm{N} 300^{\circ}$. L'anomalie interceptée par le profil

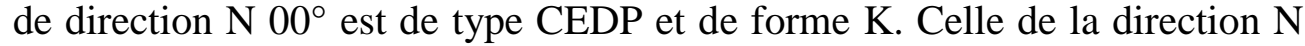
$300^{\circ}$ est de type CCL et de forme W. L'interprétation de la courbe de sondage indique trois couches et une épaisseur d'altération de 16,12m. La lithologie au niveau du forage F1 correspondant est constituée d'argile sableux, granite 
altéré humide et granite sain multi-fracturée. Sa profondeur est de $51,12 \mathrm{~m}$ avec une altération de 10,32m. Des fractures alimentées ont été recoupées de 22,92 $\mathrm{m}$ à $46 \mathrm{~m}$ produisant un débit cumulé de $7 \mathrm{~m}^{3} / \mathrm{h}$ (Figure 13a).

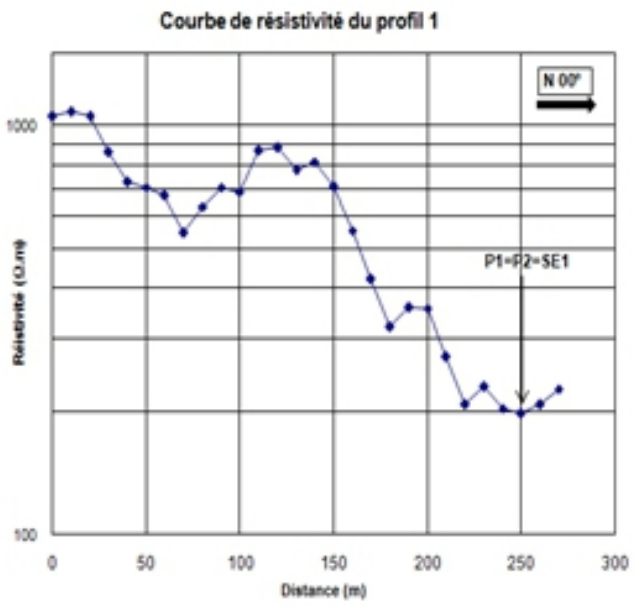

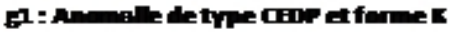

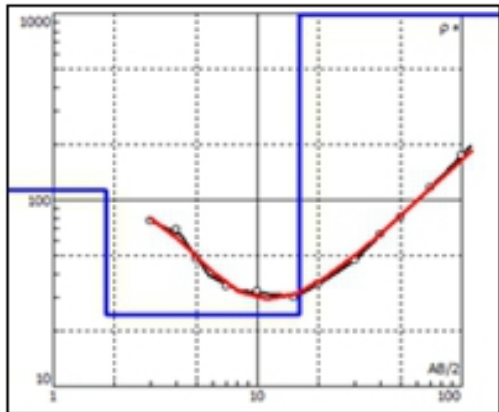

\begin{tabular}{||c|c|c|c|}
\hline N & 1 & 2 & 3 \\
\hline P & 114 & 24.3 & 31198 \\
\cline { 1 - 1 } h & 1.81 & 14.3 & \\
\cline { 1 - 1 } Alt & 1.81 & 16.1 & \\
\cline { 1 - 1 } & -1.814 & -16.12 & \\
\hline
\end{tabular}

e ESondag detpreH

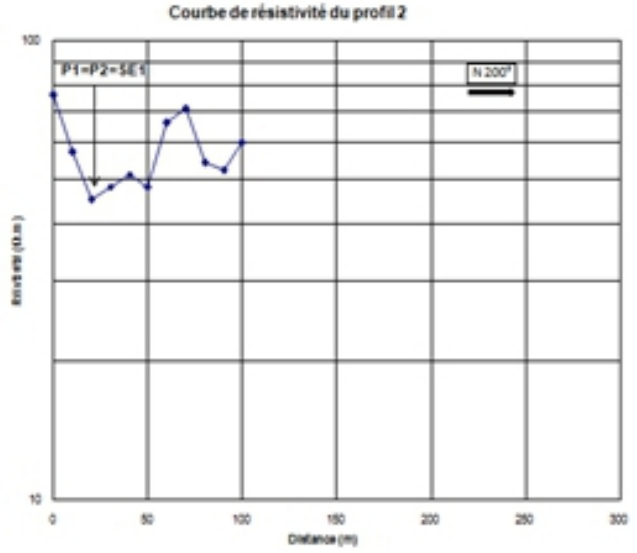

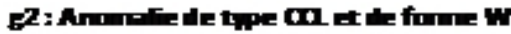

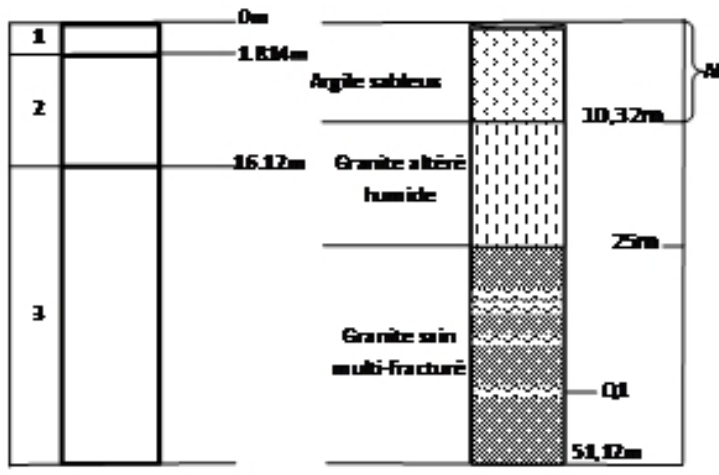

AE de 31-32;36;43m;01 $=7 \mathrm{~m}^{3} / \mathrm{h}$

OF $=7 m^{3} / \mathbf{h}$

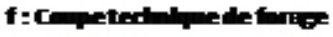

Figure 13 a: Corrélation entre les données géophysiques et les forages positifs : cas de Kouamékro

Egalement à Anatolekro, une anomalie de type CCL et de forme W est mise en évidence par un trainé de direction $\mathrm{N} 025^{\circ}$. Le sondage réalisé au droit de l'anomalie est de type A. Il est constitué de quatre couches et présente une allure ascendante sur ses trois premières couches épaisses de $36,77 \mathrm{~m}$. Cette allure connait un point d'inflexion au niveau du quatrième terrain et présente une forme concave de $40 \mathrm{~m}$ à $150 \mathrm{~m}$. Le forage exécuté au droit de cette 
anomalie a une lithologie de trois couches constitué d'argile, d'arène granitique et de granite sain multi-fracturée. Sa profondeur totale est de $73,61 \mathrm{~m}$ avec une altération de $45,92 \mathrm{~m}$. De multiples fractures productives rencontrées entre $52 \mathrm{~m}$ et $66 \mathrm{~m}$ fournissent un débit cumulatif de $4 \mathrm{~m}^{3} / \mathrm{h}$ (Figure $13 b)$.

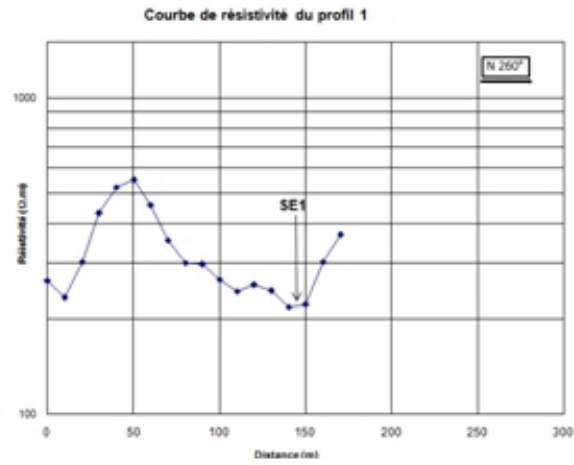

J : Anomalle de type clet forme w

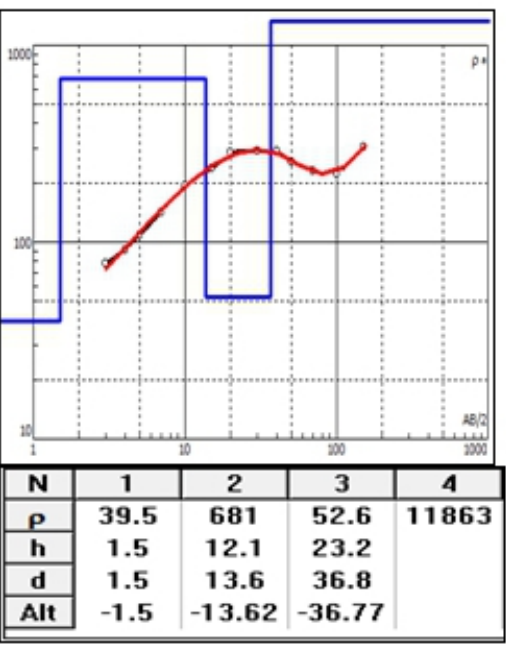

k:Sondagedetype A
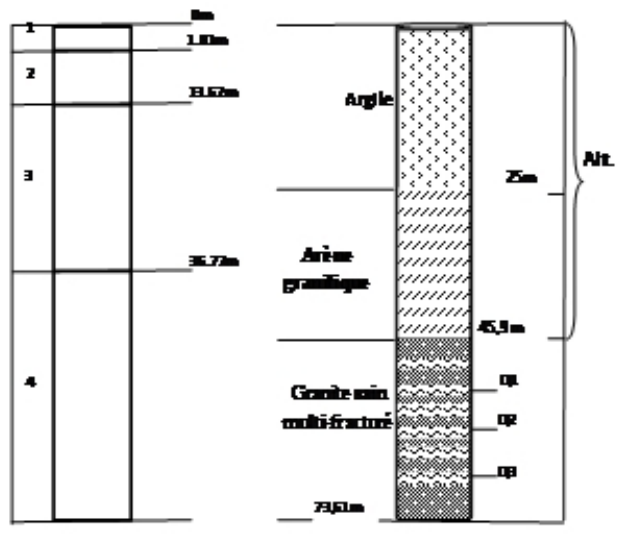

AE A $55 m$; Q1 - $23 m^{2} / h$; AE a 5 im ; CL $-3,4 m^{2} / h$

AE A EEm ; $Q 3-4 m^{2} / h$; a $73,61 m$; $Q f f-4 m^{2} / h$

I = compe techulque de forage

Figure 13 b: Corrélation entre les données géophysiques et les forages positifs : cas d'Anatolekro

\section{Discussion}

Dans la région du Haut-Sassandra, la combinaison d'un sondage de type A ou $\mathrm{H}$ obtenu sur des anomalies de type CCL et de forme $\mathrm{W}$ ou $\mathrm{U}$ a garanti un taux de succès élevé allant jusqu'à 100\%. Des indices particuliers à savoir «le fond de bateau » et «la présence d'inflexions sur les remontées finales » observés sur ces courbes de sondages seraient des indicateurs de forages productifs. En effet, des études antérieures (Biemi, 1992; Savané, 
1997, Coulibaly, 2014) ont montrés que les courbes de sondage avec « remontée trainante » type $\mathrm{A}$ et $\mathrm{H}$ ) traduisent l'influence des méga fractures dans le socle. Selon les mêmes auteurs, les inflexions sur les courbes de sondage en «branche montante » indiquent la présence de fractures. Aussi, conformément à nos résultats, Dieng et al. (2004) a t-il montré par ces travaux réalisés au Nord du Burkina Faso que les anomalies de type CCE, les anomalies de formes $\mathrm{V}$ et les sondages de type A sur des formations granitiques sont moins satisfaisantes.

Contrairement à ces résultats, Kouakou et $a l$. (2015) a montré dans ses travaux réalisés à Dabakala que la méthode géophysique donne des résultats moins satisfaisant allant jusqu'à 44,44\% de taux de succès. Selon cet auteur, ces échecs peuvent être liés à une zone fracturée présentant un pendage car certaines anomalies géologiques telles les fractures sont inclinées par rapport à la verticale. Cette différence s'explique par le fait que, dans le choix des sites, Kouakou et al. (2015) ne tient pas compte de la combinaison des indicateurs de types et formes d'anomalies et des types de sondages.

Une comparaison des données de forages avec les résultats géophysiques correspondants révèle parfois quelques différences au niveau du nombre de couches, de l'épaisseur d'altération et de l'horizon fracturé. En effet, les épaisseurs d'altération issues des interprétations géophysiques et celles obtenues du forage présentent des écarts plus ou moins importants. Ces écarts seraient dus à la différence de conception du socle qu'ont le foreur et le géophysicien (Coulibaly, 2014). En effet, du point de vue géophysique, la frange fissurée du bed-rock fait partie intégrante du niveau arénique conducteur et le socle est exclusivement constitué de la zone «compacte » résistance sous-jacente (Savadogo, 1984, Dieng et al., 2004). Par contre, le foreur observe plutôt les cuttings refoulés des forages et la vitesse d'avancement du « marteau fond de trou » pour apprécier les différentes épaisseurs / profondeurs et natures des formations géologiques traversées (Coulibaly, 2014). Ceci pourrait constituer une limite de la méthode de résistivités électriques. Toutefois, eu égard les taux de succès des implantations, elle demeure un outil appréciable pour l'optimisation de l'implantation des forages d'hydraulique villageoise en milieu de socle quand elle est bien menée.

\section{Conclusion}

Au terme de cette étude, nous retenons que la méthode de la résistivité électrique est un outil performant pour l'optimisation de l'implantation des forages d'hydrauliques villageoise en milieu de socle. En effet, dans la région du Haut-Sassandra, les types d'anomalies les plus rencontrés dans la région sont: le Compartiment Conducteur Etroit (CCE), le Compartiment Conducteur Large (CCL) et le Contact Entre Deux Palier (CEDP). En ce qui 
concerne les formes d'anomalies, ce sont les formes U, V et $\mathrm{W}$ sont les plus représentatifs. Les taux de succès correspondant à ces formes d'anomalies sont respectivement de $80 \%, 50 \%$ et $100 \%$.

Aussi, les courbes de sondages couramment rencontrés dans la zone d'étude sont-elles d'abord de type A (69\%), de type H (23\%) et enfin de type $\mathrm{KH}$ avec $(8 \%)$.

L'optimisation de l'implantation des forages par la méthode de résistivité électrique repose donc sur la combinaison d'indicateurs qualitatifs. Ainsi, une combinaison de sondages de type $\mathrm{A}$ ou $\mathrm{H}$ obtenus sur des anomalies de type CCL et de forme $\mathrm{W}$ ou $\mathrm{U}$ augure un taux de succès de forage allant jusqu'à $100 \%$. Par contre, des sondages de type A obtenus sur une anomalie de forme $\mathrm{U}$ ou $\mathrm{V}$ et de type $\mathrm{CCE}$ conduisent à des forages à taux d'échec élevé (33\% à 60\%).

\section{References:}

1. Amani K., Kouassi A., Coulibaly D., Koffi Y. et Biemi J. (2013). Application de méthodes géophysiques à l'étude de la productivité des forages d'eau en milieu cristallin: cas de la région de Toumodi (Centre de la Côte d'Ivoire).International Journal of Innovation and Applied Studies, 2 (3):324-334.

2. Ballo B. H. E. (2016). Apport de la géophysique à l'optimisation de l'implantation des forages d'hydraulique villageoise en milieu de socle : cas de la région du Haut-Sassandra (Centre-ouest de la Côte d'Ivoire). Mémoire en de Master en Sciences et Gestion de l'Environnement, Université Nangui Abrogoua, 46 p.

3. Biémi J. (1992). Contribution à l'étude géologique, hydrogéologique et par télédétection des bassins versants Sub-sahariens du socle précambrien d'Afrique de l'Ouest : Hydrostructurale, hydrochimie et isotopie des aquifères discontinus des sillons et aires granitiques de la Haute Marahoué (Côte d'Ivoire). Thèse d'Etat Université Nationale de Côte d'Ivoire, $480 \mathrm{p}$.

4. Chapellier D. $(2000 ; 2001)$. Cours online géophysique, prospection électrique de surface. Université de Lausanne ; Institut Français du pétrole, $97 \mathrm{p}$.

5. Coulibaly A. (2014). Contribution de la méthode de résistivité électrique (Traînés et Sondages électriques) à la localisation d'aquifères en zone de socle cristallin et cristallophyllien : cas de la région de Tanda, (Nord-est de la Côte d'Ivoire). Thèse unique de Doctorat, Université de Cocody, Abidjan, Côte d'Ivoire, 181p.

6. Dieng B., Kouassi H. et Bakyono A. (2004). Optimisation de l'implantation géophysique des forages en zone de socle au Nord du Burkina Faso. Sud Science et Technologie, (12):21-30. 
7. Dewandel B., Lachassagne P., Wyns R., Marechal, J-C. et Krishnamurthy N. S. (2006). A generalized 3-D geological and hydrogeological conceptual model of granite aquifers controlled by single or multiphase weathering.Journal of Hydrology, 330(1-2):260284.

8. Koffie-Bikpo C. (2013). La région du Haut-Sassandra dans la distribution des produits vivriers agricoles en Côte d'Ivoire. Revue de Géographie Tropicale et d'Environnement, (2):95-103.

9. Kouakou E.G., Lasm T., Sombo A.P.; Youan T., Baka D. et Kouadio K. E. (2015). Contribution de la géophysique à l'étude structurale et à l'identification des aquifères de fissures dans le Département de Dabakala (Centre-Nord Côte d'Ivoire). International Journal of Innovation and Applied Studies, 8(4):1752-1769.

10. Lachassagne P., Rampnoux N., Deroin J.P., Dutartre P., Laporte P., Mercier F. (1996). Compréhension de l'hydrogéologique du socle fracturé. Mise au point de méthodologies de prospection : villages des fleuves de Guyane. Rapport d'avancement du 31/12/95.BRGM/Direction de la recherche.- Rapport BRGM R39760 DR/HT 96, 41p.

11. Lachassagne P. et Wyns R. (2005). Application à la prospection et la gestion de la ressource en eau. Géosciences, (2):32-37.

12. Marescot (2009). Modélisation directe et inverse en prospection électrique sur des structures 3D complexes par la méthode des éléments finis. Thèse de doctorat ès Géosciences, Université de Nantes et de Lausanne 208p.

13. SODEXAM (2010). Société de Développement et d'Exploitation Aéroportuaire Aéronautique Météo.

14. Savadogo A. N. (1984). Géologie et hydrogéologie du socle cristallin de haute volta : Etude régionale du bassin versant de la Sissili. Thèse Doctorat ès sciences naturelles, Université de Grenoble, 350p.

15. Savane I., 1997. Contribution à l'étude géologique et hydrogéologique des aquifères discontinus du socle d'Odienné (Nord-Ouest de Côte d'Ivoire). Apport de la télédétection et d'un système d'information hydrogéologique à références spatiales (SIHRS). Thèse d'Etat èsSciences naturelles, Université de Cocody-Abidjan, Côte d'Ivoire, 398 p.

16. Wyns R., Gourry, J.C, Baltassat J.M. et Lebert F. (1999). Caractérisation multi paramètres des horizons de subsurface $(0-100 \mathrm{~m})$ en contexte de socle altéré, PANGEA 31/32 : 51-54. 